part of the paper compares changes in texture and pore size distributions. The results are $(a)$ during heat treatment a significant growth of the energetically preferred bigger pores, consuming the small ones, can be noted, $(b)$ the changes in texture increase with increasing concentration in vacancies or small vacancy clusters and internal or external stresses, $(c)$ creep in pyrocarbon leaús to a strong decrease in pore concentration of $10 \AA$ pores which in turn should lead to a decrease in creep processes. Study on matrix material and graphite: In contrast to graphite, matrix material adaitionally contains disordered, non-graphitized binder regions. In these regions an irradiation-induced graphitization takes place at irradiation temperatures far below the usual graphitization temperature. This graphitization leads to a higher order in the binder which should be perceptible by a decrease in vacancy-cluster concentration. The change of microporosity in matrix material and graphite was measured as a function of the dose of fast neutrons. The results are: The concentration of small pores is reduced in the binder by irradiation-induced graphitization. The final value is caused by a balance of two contrarily acting processes. The production of new defects during irradiation leads to a lower state of order in the material. On the other hand, the degree of order increases by the use of irradiation-produced Frenkel defects for atomistic transport processes. Therefore in the low-ordered binder an irradiation-induced graphitization is predominant at low doses. With increasing dose and order an increasing disordering process is superimposed until an equilibrium is reached. The concentration of vacancy clusters therefore decreases to a limiting value. Unlike in matrix material, the order in graphite is reduced; the concentration of vacancy clusters increases from the beginning of the irradiation.

J. Appl. Cryst. (1974). 7, 224

\title{
Cluster Arrangement of Point Defects in $\gamma$-Irradiated LiF
}

\author{
By W. Wagner, W. Waidelich AND O. WOHOFSKY \\ Physikalisches Institut der Technischen Hochschule Darmstadt and Physik-Department der Technischen Universität \\ München, German!. (BRD)
}

The clustering of point defects in $\gamma$-irradiated LiF single crystals was investigated by measuring the small-angle X-ray scattering after various $\gamma$ doses. The curves were obtained by means of a Kratky camera on a $30 \mathrm{~kW} X$-ray generator with rotating anode. With increasing irradiation time the scattered intensity shows a maximum at $10^{-2} \AA^{-1}$. It is suggested that this maximum is due to a regular arrangement of the defect clusters. The mean distance is $500 \AA$.

J. Appl. Cryst. (1974). 7, 224

\section{Inhomogeneities in Molten Alloys}

\author{
By S. STEEB \\ MPI-Sondermetalle, 7000 Stuttgart-1, Seestrasse 92, Germany. (BRD)
}

\begin{abstract}
Molten alloys consisting of two components under thermodynamic-equilitrium conditions can be classified into one of three groups: compound, segregation and solubility grours. This classification can best be made by using diffraction methods. In compound-forming alloys often agglomerates are detected. i.e. regions with a structure similar to that of the intermetallic compound at the corresponding concentration. Agglomerates only show small differences in electron density or density of scattering length versus the matrix and therefore neither from small-angle X-ray scattering nor from small-angle neutron scattering can the shape and magnitude of the agglomerates be determined. Within segregation melts, on the other hand, regions can exist which contain atoms of one kind only, and which are called concentration fluctuations or clusters. In the author's laboratory to date melts from the following systems have been investigated by SAXS and SANS: In- $\mathrm{Zn}, \mathrm{Bi}-\mathrm{Cu}, \mathrm{Ag}-\mathrm{Pb}, \mathrm{Cd}-\mathrm{Ga}, \mathrm{Bi}-\mathrm{Ga}, \mathrm{Al}-\mathrm{Sn}, \mathrm{Al}-\mathrm{In}, \mathrm{Al}-\mathrm{Zn}$, $\mathrm{Mg}-\mathrm{In}, \mathrm{Al}-\mathrm{Ga}, \mathrm{Ag}-\mathrm{Al}$ and $\mathrm{Cu}-\mathrm{Sb}$. A SAS intensity which surmounts the contribution of density fluctuations and therefore can be ascribed to concentration fluctuations was found with melts from the systemis $\mathrm{Al}-\mathrm{Sn}, \mathrm{Al}-\mathrm{In}$ and $\mathrm{Cu}-\mathrm{Bi}$. From Guinier plots particle diameters were obtained of about $12 \AA$, i.e. the inhomogeneities or clusters contain one coordination sphere only. Concerning the structural aspects of these melts, the observed inhomogeneities are identical with the embryos or nuclei necessary for the understanding of solidification. Of course, the run of intensity can also be interpreted with the aid of an Ornstein-Zernike plot which finally yields a correlation length.
\end{abstract}

